\title{
An apparatus for quantitative high-harmonic generation spectroscopy in molecular vapours
}

Felicity McGrath, ${ }^{1, \text { a) }}$ Allan S. Johnson, ${ }^{1, a)}$ Dane R. Austin, ${ }^{1}$ Peter Hawkins, ${ }^{1, b)}$ David Wood, ${ }^{1}$ Lukas Miseikis, ${ }^{1}$ Emma R. Simpson, ${ }^{1,}$ c) Marta Castillejo, ${ }^{2}$ Ricardo Torres, ${ }^{1}$ Susan Parker, ${ }^{1}$ Thomas Siegel, ${ }^{1}$ and Jon P. Marangos ${ }^{1}$

${ }^{1)}$ Blackett Laboratory, Imperial College London, Prince Consort Road, London, SW7 $2 A Z$

${ }^{2)}$ Instituto de Quimica Fisica Rocasolano, Spanish National Research Council (CSIC), Serrano 119, 28006 Madrid, Spain

(Dated: 30 May 2017)

We present an apparatus for performing gas phase high-harmonic generation spectroscopy of molecules primarily found in the liquid phase. Liquid molecular samples are heated in a temperature controlled bath and their vapour is used to back a continuous flow gas jet, with vapour pressures of over 1 bar possible. In order to demonstrate the system, we perform high harmonic spectroscopy experiments in benzene with a $1.8 \mu \mathrm{m}$ driving field. Using the unique capabilities of the system, we obtain spectra that are nearly free from the effects of longitudinal phase-matching, amenable to comparison with advanced numerical modelling.

PACS numbers: 07.57.-c

\section{INTRODUCTION}

The measurement of ultrafast dynamics in atomic and molecular systems is a topic of great interest in modern laser physics, and a primary goal of attosecond science $^{1}$. An understanding of electronic and nuclear dynamics at the few-femtosecond to attosecond time scale is key to advancing the understanding of chemical reactions ${ }^{2}$, the development of petahertz electronics 3 , and interpreting processes such as photosynthesis to facilitate their artificial development. Techniques such as Attosecond Transient Absorption Spectroscopy (ATAS) ${ }^{4}$, Time-Resolved Photoelectron Spectroscopy (TR-PES) ${ }^{5}$, Coulomb Explosion Imaging (CEI) ${ }^{6 / 7}$, and High Harmonic Generation Spectroscopy (HHGS $)^{8}$ allow attosecond dynamics to be observed directly in the time domain. These techniques can be generally devided into photoion/photoelectron techniques (CEI, TR-PES) and optical techniques (ATAS, HHGS). Photoion/photoelectron techniques benefit from high collection efficiencies and require only very low sample densities, but require ultra-high vacuum conditions, complex apparatus, long integration times, and are limited to relatively small molecules. All-optical techniques conversely require much higher sample densities, but use comparatively simple apparatus and can be performed in lower vacuum conditions.

The systems necessary for generating the isolated and synchronized attosecond pulses required for ATAS and TR-PES are generally extremely complex $9 \mid 10$. HHGS offers an alternative route towards attosecond measurements which does not require attosecond pulses; instead, in HHGS, the sample under study is exposed to a high-intensity infrared laser pulse and emits high-order harmonics of infrared laser frequency. The harmonic spectrum carries information on the nuclear and electronic structure, as well

\footnotetext{
a) These authors contributed equally to this work.

b) Current address: Department of Physics, University of Marburg, 35032 Marburg, Germany.

c) Current address: Department of Physics, Lund University, PO Box 118, SE-221 00 Lund, Sweden.
}

as naturally encoding a sub-femtosecond resolution pumpprobe measurement of cation dynamics 811 , allowing for true attosecond measurements in a simple and compact setup. Recent advances in HHGS measurements include the tracking of structural changes in the electron shells of halogenated methanes $\frac{15}{15}$ and resolving chiral dynamics on electronic timescales 16 .

Most HHGS measurements have thus far been performed with molecules that are in the gas phase at room temperature and pressure, as gas targets allow easy switching between atomic or molecular species external to the vacuum chamber, are naturally replenish-able, and allow for the study of targets free from the influence of the environment. The most significant drawback is that only a limited number of small molecules occur naturally in the gas phase, which limits the scope of these techniques; they have yet to be applied to studying biologically relevant molecules such as peptides, DNA bases, and carcinogens. Two potential avenues for advancing HHGS to more complex systems include solid targets and liquid jets/droplet injectors. Both liquid and solid phase targets automatically have the high densities required for optical measurements, but come with separate drawbacks. In both cases the high densities actually necessitate very thin sample to reduce phase-matching effects. For solid targets this means creating very thin films, which need to be free-standing for use with HHGS. Additionally, either very long wavelengths are necessary to operate at lower intensities ${ }^{17}$ or the sample is destroyed each shot and needs to be replenished. Generating liquid phase targets with a few microns thickness also presents significant challenges, requiring complex experimental apparatus and is generally limited to liquids with low surface tension. Most importantly, the vapour pressure of the liquid must be low enough that the vapour phase around the liquid sample contributes only negligibly to the overall signa $\sqrt{18}$.

In order to take advantage of the many benefits of gas phase HHGS, we have focused on developing the capability to introduce larger molecules into the gas phase at sufficiently high densities for optical measurements. In many cases biologically relevant molecules exist in the liquid phase 
at room temperature and pressure, but their vapours can form a low density target for laser interactions ${ }^{2}$. While for particle detection low gas densities are acceptable due to the high detection efficiency, for all-optical methods significantly higher densities are required. In general densities in a $1 \mathrm{~mm}$ long interaction region of $>10^{17}$ molecules $/ \mathrm{cm}^{3}$ are necessary for gas phase spectroscopy, which places much more stringent demands on any system supplying vapour phase samples. The vapour pressure can be increased by raising the temperature of the sample, suggesting a route towards providing the necessary densities.

As with nonlinear photo-particle methods, in HHGS one must account for the effect of the spatial distribution of the laser over the interaction volume. However HHGS has the additional complication that the signal is a coherent superposition rather than a simple average, leading to significant phase-matching effects ${ }^{19 \mid 20}$ which reshape the single emitter response. In principle, all of these effects can be described theoretically and incorporated into the processing of HHGS data. However, advanced single-emitter theories of HHG in molecules are often sufficiently computationally intensive to prohibit full macroscopic integration, and in any case, it is advantageous to simplify the analysis as much as possible. One approach is to to eliminate or reduce coherent macroscopic averaging is to ensure the target is well described by a thin sheet of atoms with negligible longitudinal phase matching, harmonic absorption, and reshaping of the laser pulse due to propagation effects. In this thin medium regime, quantitative theoretical modelling requires macroscopic integration over only the transverse plane with a well-defined laser profile, and systematic adjustment of laser parameters such as the intensity or wavelength do not introduce complicating factors due to reshaping of the laser field. The thin medium regime places upper bounds on both the length and the density-length product of the target.

Here we present an apparatus capable of introducing small organic molecules $(\approx 100$ a.m.u.) into the gas phase for HHGS experiments. Our system is based on a heated sample bath using vapour pressure to introduce volatile molecules into the gas phase at pressures greater than 1 bar, together with a heated gas delivery system to introduce the molecular vapour into vacuum. The density achieved is sufficient to obtain a precise HHGS signal with a target depth of $\approx 500 \mu \mathrm{m}$, placing us within the thin medium regime. In order to demonstrate our system, we perform HHGS measurements on substituted benzene molecules, which naturally occur in the liquid phase. Substituted benzenes have low ionization potentials (less than $9.2 \mathrm{eV}$ ) and thus are best addressed by long wavelength fields, and so we drive HHG using a $1.8 \mu \mathrm{m}$ wavelength driving laser. Benzene has a high degree of symmetry, and the localized $\pi$ electron environment can be probed by substitutions of halogen or methyl groups on the ring. Benzene is also a fundamental building block of many biologically relevant molecules and is the main structural component of many isomeric and chiral molecules, and is thus an ideal stepping stone to HHGS of even more complex molecules.

The paper is structured as follows. Section [II presents the specifications and important considerations in the design of the apparatus, as well as the apparatus itself. Sec- tion III presents a variety of experimental evidence that we can successfully obtain an experimental signal which is minimally distorted by macroscopic effects. Our results shows the robustness and reproducibility of carefully acquired HHGS measurements, and that the data obtained can provide insight into molecular dynamics in a range of organic molecules. Section IV] concludes.

\section{APPARATUS AND EXPERIMENTAL DESIGN}

The propagation of the driving laser beam through focus naturally imposes a longitudinal variation in both intensity and phase. The thin medium limit thus corresponds to a medium with longitudinal extent significantly less than the Rayleigh range, which for most HHG laser systems implies a medium length below $1 \mathrm{~mm}^{21}$, with even shorter lengths desirable. This precludes the use of gas-cells (finite or semi-infinite) and suggest the use of gas jets, where the longitudinal extend of the source is determined by the jet aperture, which can be tens of microns in diameter. The challenge is then to deliver sample with high enough backing pressure to the jet; unlike gas cells, which can generate harmonics with pressures of a few tens of millibars, achieving sufficiency densities with gas jets requires backing gas pressures on the order of 1 bar.

Having identified gas jets as the preferred geometry for quantitative HHGS, we briefly describe previous work on the vapour delivery configurations for HHGS. Previous experiments studying liquid phase molecules have utilised heated pulsed-valve gas jets or gas cells for introducing the sample to the interaction region. Hay et al. ${ }^{[22}$ implemented a heated pulsed-valve gas jet with the sample housed in a small crucible within the vacuum chamber. The limitations of this design include the inability to switch between samples, the small capacity $(\approx 5 \mathrm{~mL}$ ) of the sample crucible limiting measurement time, and the operating frequency of the valve, limited to $10 \mathrm{~Hz}$. In contrast, commercial CPA laser systems typically operate at $1 \mathrm{KHz}$ or higher repetition rates. These issues are partially mitigated by the development of the Even-Lavie pulsed-valve gas jet, which can operate at repetition rates of up to $1 \mathrm{kHz}^{23}$. In addition, this design of valve has a typical opening time of $30 \mu \mathrm{s}$ which facilitates expansion conditions providing rotational cooling 24 . These valves are, however significantly more expensive and complex than gas jets or cells, and may lead to strong cluster formation in molecules. Furthermore corrosive molecules at elevated temperatures have been found to damage the internal components of pulsed valves 25 .

We developed a system based around a continuous flow gas jet $(200 \mu \mathrm{m}$ diameter orifice) for the intended HHGS experiments in vapour phase molecules, shown in Figure 1 . This allows us to have medium length significantly shorter than the Rayleigh range for reasonable laser parameters, and to suppress longitudinal phase-matching effects. A continuous flow jet has been chosen as it presents numerous advantages over the other previously used vapour sources. A continuous flow jet allows each incident laser pulse to interact with the medium and circumvents the need for having an apparatus to synchronize the laser shots with 


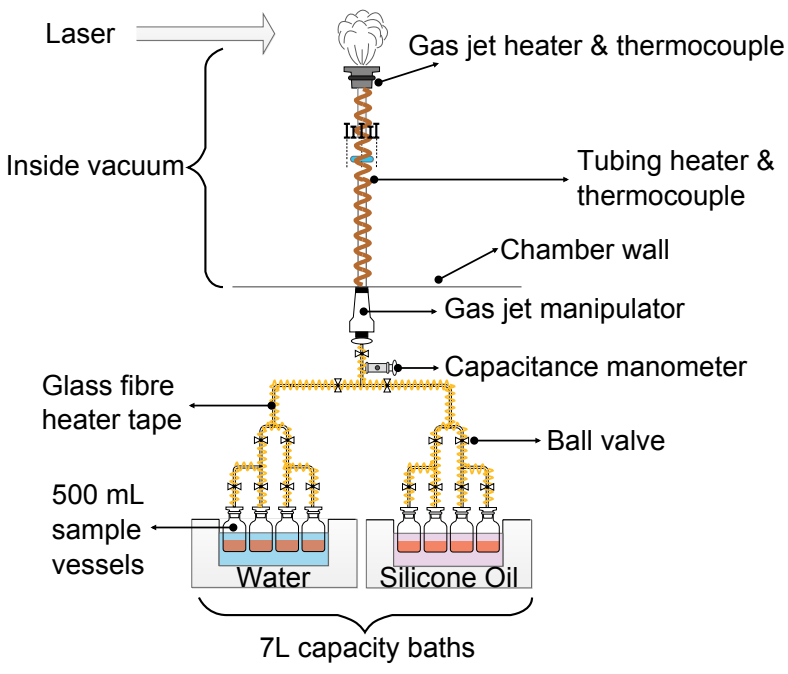

FIG. 1: Full vapour delivery apparatus. The apparatus consists of 4 stages: sample heating baths, outside vacuum vapour manifold, in-vacuum vapour delivery, and continuous flow jet. Each stage is elaborated upon in the text.

the opening time of the valve poppet. Furthermore, the static flow significantly decreases the likelihood of clogging, reduces cooling and thus cluster formation 26 , and allows for simple switching of samples externally by changing the gas reservoir upstream. This design is also simple and inexpensive in comparison to the other delivery methods available, can be heated to over $600^{\circ} \mathrm{C}$, and the gas jet can be made extremely thin simply through the size of aperture used. A continuous flow jet can also be used in molecular alignment experiments provided that the jet construction fulfils the conditions for obtaining a supersonic expansion 27 .

In order to perform HHGS in molecules that exist in the liquid phase at room temperature, the gas system must be carefully controlled to ensure that a precise and stable density of molecules is achieved in the interaction region. This includes not only the final gas jet, but the sample reservoirs, vapour delivery, and heat control, especially as many of the molecules are corrosive or reactive to other materials. The complete apparatus is shown in Figure 1, and consists of two heated liquid baths each holding four sample vessels, connected to a heated gas manifold for delivery to a heated continuous flow gas jet. Each of these sub-systems is elaborated upon in the following discussion. A capacitance manometer (MKS Instruments) is used to measure the backing pressure before the tubing enters the vacuum chamber, which has the advantage of giving accurate pressure readings independent of gas species. The pressure is logged at $1 \mathrm{~s}$ intervals to ensure stable operation throughout measurements.

The vapour generation stage consists of two 7 litre temperature-stabilized heated-liquid baths, each of which can host four $500 \mathrm{~mL}$ sample vessels. The large total volume relative to the sample volume allows for stable vapour pressures. One bath holds deionized water (Memmert WNB 7 ) and is used to heat any molecules for which $\approx 1$ bar of vapour pressure can be generated at temperatures $\leq 100^{\circ} \mathrm{C}$. Examples of such molecules include benzene, toluene, water, and fluorobenzene. Any molecules which require temperatures in excess of $100^{\circ} \mathrm{C}$ to produce vapour pressures $\approx 1$ bar (i.e. xylene isomers, halogenated benzenes) are heated in a silicone oil bath (Memmert ONE 7), with a maximum temperature of $200^{\circ} \mathrm{C}$. The temperature stability of each bath is better than $0.1^{\circ} \mathrm{C}$, allowing us to precisely control the vapour pressure via the sample temperature. This allows us to dispense with regulators for controlling the backing pressure of the gas jet, which are highly problematic at high temperatures and with corrosive molecules. In order to verify this technique, we compare the behaviour of our system to the the Clausius-Clapeyron equation, which describes the relationship between the temperature of a liquid and its vapour pressure:

$$
\ln \frac{P_{2}}{P_{1}}=\frac{\Delta H_{v a p}}{R}\left(\frac{1}{T_{1}}-\frac{1}{T_{2}}\right)
$$

where $P_{1}$ is the partial pressure of the liquid at $T_{1}, P_{2}$ is the partial pressure of the liquid at $T_{2}, R=8.3145 \mathrm{~J} / \mathrm{mol} / \mathrm{K}$ is the ideal gas constant and $\Delta H_{v a p}$ is the enthalpy of vapourization in units of $\mathrm{J} / \mathrm{mol}$. Figure 2 shows experimentally measured pressure-temperature curves in benzene as well as the expected curve from the Clausius-Clapeyron equation using the tabulated enthalpy. The systematic deviation may be attributed to the gas conductivity of our gas manifold, as the temperature is measured at the bath while the pressure is measured close to the gas jet. Nevertheless, the correct scaling validates our approach and allows for precise control of the backing pressure.

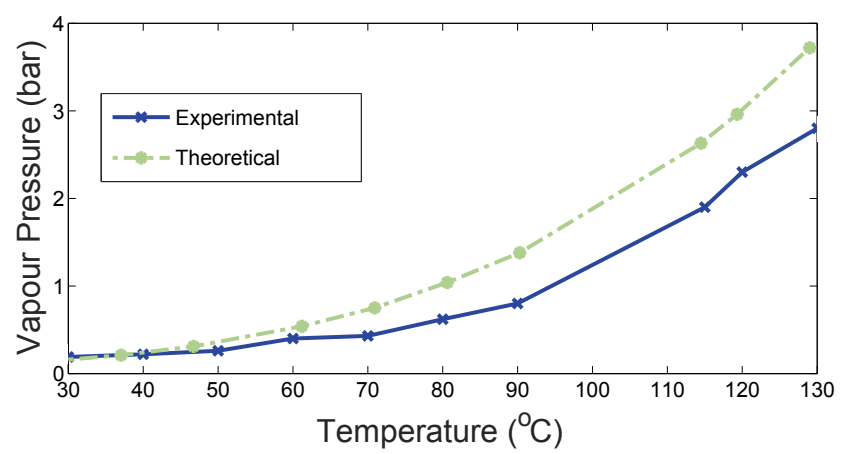

FIG. 2: Pressure-temperature dependence of benzene samples measured in our system using a capacitance manometer, and calculated using the Clausius-Clapeyron equation.

The vapour delivery manifold is constructed such that switching between samples is rapid. This design is distinguished from previous systems in that sample switching is performed externally to the vacuum chamber which houses the jet. Each sample can be isolated from the manifold and removed without breaking vacuum or disturbing the apparatus. We can also use larger sample vessels as they are not contained within the vacuum system itself, further reducing downtime for sample switching and allowing longer measurements. The entire manifold is constructed from stainless steel. A fibreglass insulated heating tape (Hemi Heating HTS range) is used to heat the lines to prevent the condensation of any vapour, which would otherwise contribute to sample contamination and create the potential 
for blockages. The tubing from the reservoirs to the jet is necessarily mounted from the bottom of the chamber such that the vapour works against gravity. This is to prevent any debris or condensation on the inner walls of the tubing falling into and blocking the gas jet. Any residual condensation is removed from the lines during the sample switching procedure, in which the system is evacuated, then purged with dry nitrogen.

Heating the system inside the vacuum chamber presents additional challenges, for both the gas delivery and the jet. A silicon rubber heater (Watlow Ltd.) was used to heat the tubing. The design provided full coverage of the tubing, which is necessary as stainless steel is a poor conductor of heat. A layer of Kapton tape between the tubing and the heating element electrically isolates the heater from the tubing. A thermocouple was placed midway up the tubing before the jet unit to control the temperature of the tubing. The silicon rubber heater is capable of heating to greater than $200^{\circ} \mathrm{C}$, and was kept at $150{ }^{\circ} \mathrm{C}$. For the continuous flow gas jet we used a Swagelok plug with a 200 $\mu \mathrm{m}$ diameter aperture drilled through it. A 1 inch diameter mineral insulated band heater (Watlow MB1A1AN4) was used to heat the nozzle. A brass jacket was used to establish thermal contact between the heater and the jet. The thermocouple for the gas jet fastens to the brass jacket 2 $\mathrm{mm}$ away from the gas jet. This unit allows the jet to be heated to $600^{\circ} \mathrm{C}$, but it was held at $200^{\circ} \mathrm{C}$. Both heating elements (tubing and jet) are controlled independently by a 2 channel dual loop PID controller (Watlow EZ-ZONE). The stability of both heating elements is $\pm 0.1^{\circ} \mathrm{C}$.

Sample vessels were typically refilled every three days; switching between samples took less than two minutes. Steady flow was observed for almost all molecules at all pressures, with a backing pressure stability of $0.3 \%$ over several hours in benzene. The exception was fluoro-benzene, which was observed to condense even in the presence of the high temperatures and cause sputtering; we speculate this is due to it being more strongly polar. It is possible that a larger jet aperture and higher temperatures would overcome this problem; alternatively a dilute carrier gas could be added. Despite this limitation, the extremely stable backing pressure stability of all other vapours, the long time between sample refills, and the rapid changing of species allows us to perform highly systematic studies of HHGS from small molecular vapours as described in the next section.

\section{EXPERIMENTAL RESULTS}

Our laser system (Figure 3) consists of a commercial optical parametric amplifier (HE:TOPAS, Light Conversion) pumped by $8 \mathrm{~mJ}, 25 \mathrm{fs}, 800 \mathrm{~nm}$ pulses at a $1 \mathrm{kHz}$ repetition rate from a commercial Titanium Sapphire chirped pulse amplification laser (Red Dragon, KMLabs). We take the idler from the OPA, centered at $1800 \mathrm{~nm}$, and couple it into a $400 \mu \mathrm{m}$ diameter hollow capillary for spatial filtering. Using a second harmonic FROG we measure the pulse duration at output to be $30 \mathrm{fs}$, with a pulse energy of $600 \mu \mathrm{J}$ in the HM11 mode. We collimate the output of the hollow capillary using a $75 \mathrm{~cm}$ focal length mirror. We focus the pulses using an anti-reflection coated $50 \mathrm{~cm}$ focal length $\mathrm{CaF}_{2}$ lens to a spot size of $170 \mu \mathrm{m}$, with Rayleigh range $>10 \mathrm{~mm}$. The lens is mounted on a motorized translation stage (Z25M/1 Thorlabs) for scans of the focus z-position relative to the jet. A motorized iris before the lens allows for intensity scans; an iris is preferable to a waveplate-polarizer as the drop in intensity is somewhat compensated by the larger focal spot, leading to observable signals over a larger intensity range. A $1 \mathrm{~mm} \mathrm{CaF}_{2}$ pickoff is used to record the focal size and pulse energy of each acquisition. The laser passes through a $\mathrm{CaF}_{2}$ window approximately $10 \mathrm{~cm}$ after the lens into a high vacuum chamber containing the heated gas jet. The emitted harmonics and residual fundamental then pass through the differential pumping into the spectrometer chamber. In the spectrometer chamber the harmonics pass through a motorized filter wheel (Thorlabs) containing thin metallic filters. The harmonics then illuminate a variable-line-space flat-field grating (Hitachi 001-0437) and are dispersed and focused on an CsI coated microchannel plate and phosphor screen assembly (Photonis). The harmonic spectrum on the posphor screen is recorded using an objective lens (25 mm FL, f/0.95) in conjunction with a low noise camera (PCO Pixelfly). The grating is fully manipulable under vacuum to optimize the harmonic focusing and resolution. We calibrate the spectrometer using aluminium absorption edges at 20 and $70 \mathrm{eV}$.

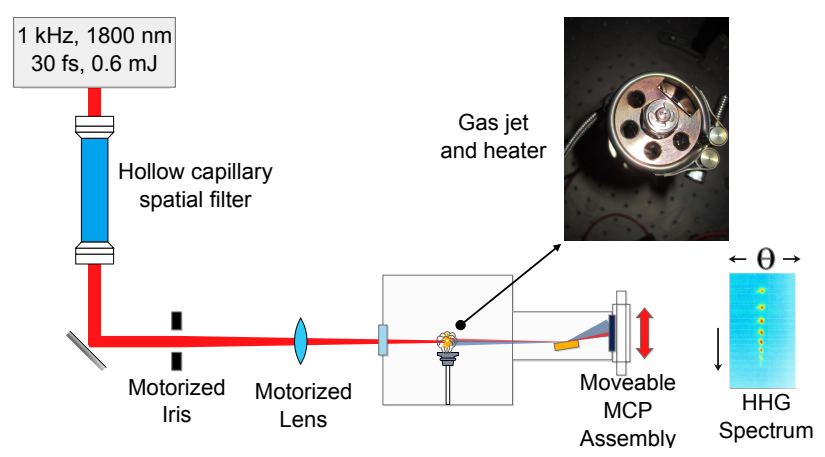

FIG. 3: Optical beamline and XUV spectrometer setup for HHGS from benzene vapors. Further details are provided in the text.

Figure 4 shows harmonic spectra obtained in benzene, toluene, $m$-xylene and $p$-xylene at an intensity of $35 \mathrm{TW} / \mathrm{cm}^{2}$. All exhibit good signal to noise, good harmonic resolution and contrast, and high spatial uniformity with low divergence. Similar spectra have been obtained in deuterated-benzene and $o$-xylene, as well as chlorine, iodine, and bromine substituted benzenes. We have not found any signatures of long trajectory contributions due to their larger divergence, which reduces their at-detector flux to below our sensitivity. There are significant differences between the four spectra shown in Fig. 4, and these are currently the subject of further analysis and theoretical investigation. Our aim here is to establish that these harmonics are produced in a thin medium regime, enabling straightforward comparison with theory across a range of laser intensities.

We first ascertained the effective target thickness by monitoring the intensity of the harmonics as the target was laterally translated across the beam and assuming the jet is rotationally symmetric; a thickness of $500 \mu \mathrm{m}$ was obtained in this way. Calculations show that for our focal length, the 
geometric phase shift of the fundamental and intensity dependent dipole phase shift over the extent of the medium is $\approx 680 \mathrm{mrad}$ for $40 \mathrm{eV}$ harmonics, the highest energy we observe.

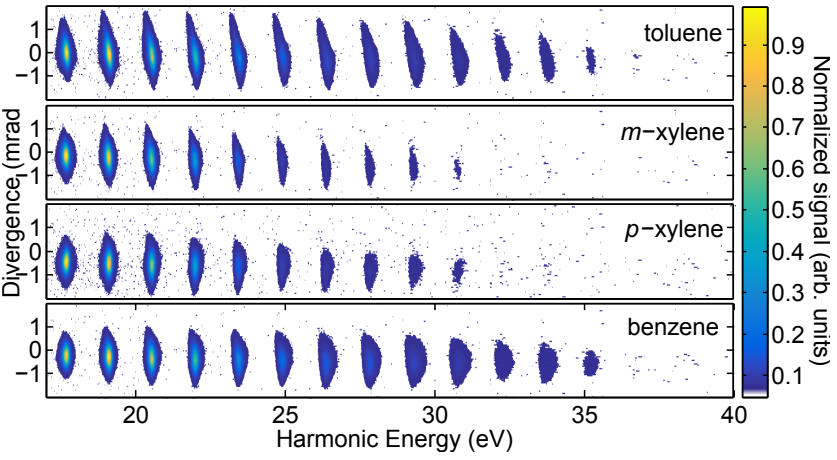

FIG. 4: Sample harmonic spectra from benzene, toluene, $m$-xylene and $p$-xylene at a driving intensity of 35 $\mathrm{TW} / \mathrm{cm}^{2}$. Each exhibits well defined harmonics with clean spatial and spectral structure, suggesting limited plasma generation.

Having established that the target is sufficiently thin, we now turn to its density length product. We take an empirical approach: we measure harmonic spectra over a range of target densities, and test for violations of the self-similar scaling law expected from phase-matched coherent build-up in the absence of absorption

$$
I(\omega, \rho)=A \rho^{2} \bar{I}(\omega)
$$

where $\omega$ is the harmonic frequency, $\rho$ is the density, for which we use the backing pressure as a proxy, $I(\omega, \rho)$ is density-dependent harmonic spectrum, $A$ is a constant, and $\bar{I}(\omega)$ is the spectrum at reference pressure. Any violation of the thin-medium assumptions would lead to violations of (2). Harmonic absorption would reduce the $\rho^{2}$ scaling and also reshape the spectrum as it is frequency dependent. If phase matching were significantly influenced by a neutral gas or plasma contribution, then adjusting the pressure would have a similar effect. Linear and nonlinear reshaping of the laser would also, if present, be affected by a change in pressure. Clustering, expected to increase dramatically with pressure, would also alter the harmonic spectrum 28 .

Figure 5a) shows the growth of the sum of the 23rd, 25th, and 27 th harmonics of benzene as a function of backing pressure over a range from 0.025 to 0.25 bar. We obtain an excellent power-law fit, with slightly super-quadratic behaviour $\left(P^{2.3}\right)$ where $P$ is the backing pressure. We attribute the super-quadratic growth to dynamics of the gas jet, leading to a slightly nonlinear relationship between $P$ and $\rho$. Figure $5 \mathrm{~b}$ ), in which spectra taken in benzene at four different backing pressures of up to 0.32 bar are normalized to the integrated signal level. The variations between the spectra are within the reproducibility of the measurement, as shown in Figure 5;) where the differences in peak height from the mean are plotted and show no systematic trends, and hence within this range we can safely use the highest backing pressure. For further increases in backing pressure we begin to observe significant distortions to the spectral shape, indicating the onset of absorption and phase-mismatch, and hence perform subsequent measurements at a backing pressure of 0.3 bar.
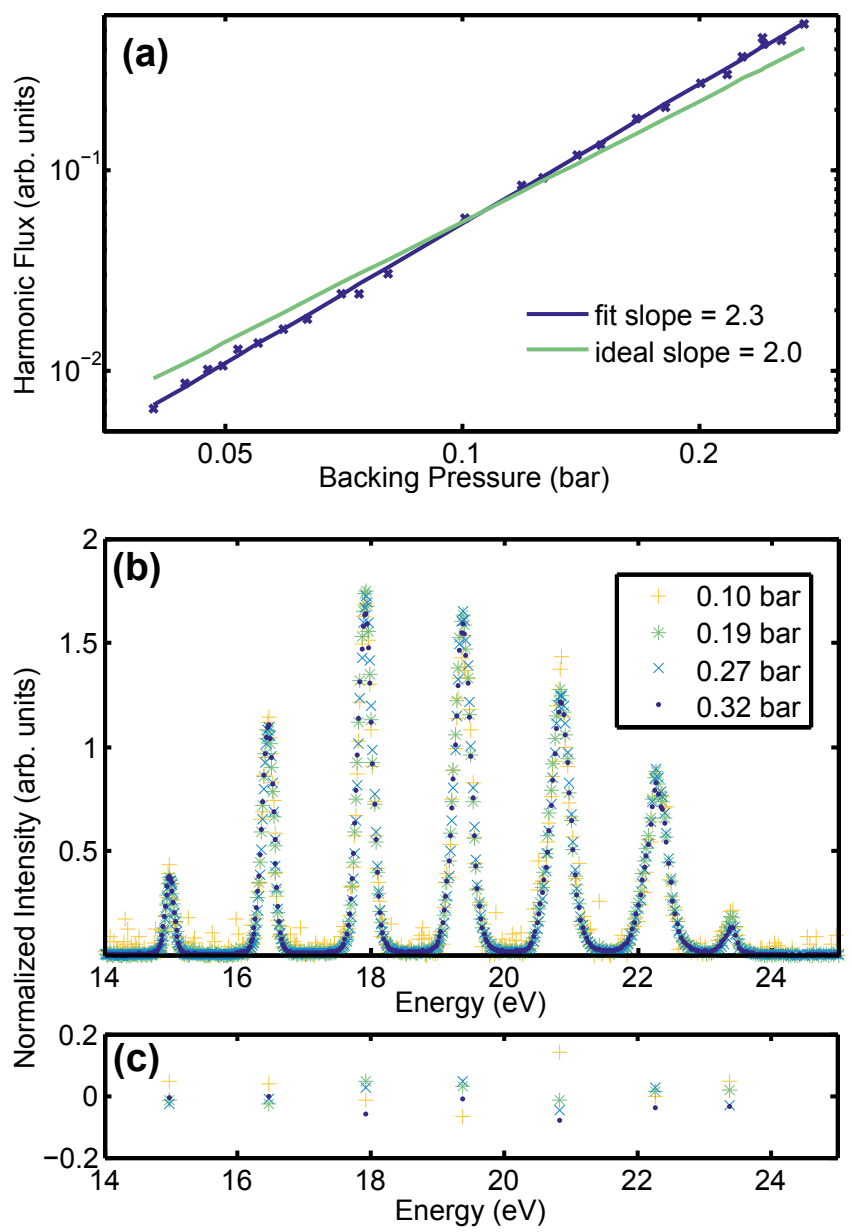

FIG. 5: a) Total flux of harmonics 23, 25, and 27 generated in benzene as a function of backing pressure. The ideal phasematching growth is given by the dotted green line; the deviation between the two is attributed to gas jet dynamics. No effects of saturation can be seen. b) The change in harmonic spectra from benzene as a function of backing pressure. Each spectra is normalized to the total harmonic signal. c) Difference in harmonic peak heights as a function of backing pressure from the mean spectrum averaged over all backing pressures. No significant or systematic change in harmonic shape is visible over a large range of backing pressures.

It is possible to saturate the harmonic response through overdriving the system in question and causing excessive ionization. This can both distort the macroscopic response through plasma generation and directly saturate the single emitter through ground state depletion. To ensure such effects are not significant we perform intensity scans looking for the characteristic signature of single emitter HHG, the cutoff energy scaling law $E=3.17 U_{p}+I_{p}$. Figure 6 shows total signal normalized spectra as a function of intensity. Up to intensities of around $30 \mathrm{TW} / \mathrm{cm}^{2}$ the harmonic cutoff monotonically increases with increasing intensity, before saturating and even decreasing as the system is over-driven. Furthermore, over this range the cutoff scaling provides a precise measure of the peak intensity. Note this saturation 
takes place before the appearance of the harmonic frequency blue shifting from the generation of plasma, which can be observed at higher powers.

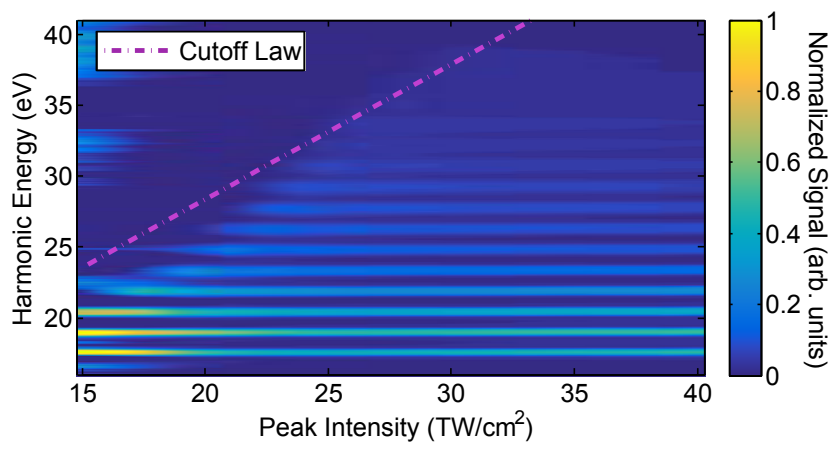

FIG. 6: Spatially integrated harmonic spectrum from benzene as a function of iris diameter (intensity). The spectrum at each intensity has been normalized by its area.

The smooth motion of the cut-off for smaller intensities shows the classical cut-off behaviour, while at higher intensities ionization saturation becomes significant.

Summarising, our pressure scan shows that the spectrum is not distorted by absorption, phase-matching, or clustering, and the intensity scan reveals the point at which excessive ionization distorts the spectrum. These scans are time-consuming, but our results show little variation in the optimal conditions across the full range of molecules we delivered to the system. Figure 7 shows the harmonic amplitudes from benzene under nominally identical conditions taken at seven different times across a period of two weeks. The spectra show good repeatability, suggesting that these control scans need only be performed once. This long term stability is also critical for any experiment that aims to perform comparative scans with respect to additional parameters (intensity, ellipticity, wavelength) or compare spectra of different molecules. Our system thus demonstrates the potential for truly systematic comparisons of the HHG spectra from different species or with regards to external parameters, under conditions satisfying the thin medium approximation.

\section{CONCLUSIONS}

In order to extend HHGS to larger molecules which do not normally exist in the gas phase, we have developed an apparatus capable of producing a stable high pressure vapour from a range of molecules existing in the liquid phase at room temperature. We back a thin $(200 \mu \mathrm{m})$ diameter nozzle to form a thin gas jet from which we generate harmonics, in which the HHG process is well described by a thin medium approximation. The utility of the apparatus is not limited to HHGS, but can be used for any optical ultrafast spectroscopy method which requires high gas densities over a confined longitudinal region, for instance time-resolved X-ray absorption studies.

We have acquired HHG spectra in low $I_{p}(\leq 10 \mathrm{eV})$ organic molecules using a $30 \mathrm{fs}, 1800 \mathrm{~nm}$ laser source. HHG spectra have been successfully and reproducibly acquired in benzene, methylated benzenes, halogenated benzenes, and deuterated

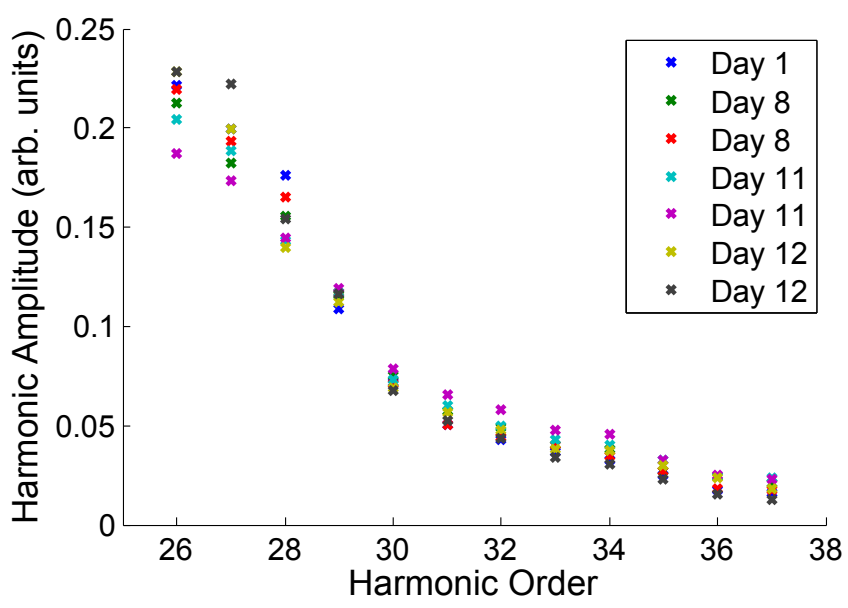

FIG. 7: Harmonic amplitude of benzene (normalized to total yield) taken at seven different occasions across a period of two weeks. The repeatability of the spectrum is good and shows the reproducibility of spectra over long time periods.

benzene, demonstrating the capability of this apparatus to switch reliably between up to 8 different samples quickly and non-invasive of the apparatus. This is a necessary requirement for experiments involving the comparison of spectra under similar generating conditions. Careful scanning of macroscopic conditions has identified a range of laser intensities and backing pressures in which sufficient high-harmonic signal is generated but the thin medium approximation is satisfied and the response is not saturated. The spectra can be quantitatively compared to theoretical calculations that involve integration over the transverse dimensions only, a significant simplification compared to full propagation. The design of the apparatus enables highly stable, reproducible spectra to be acquired from a wide range of molecules and facilitates simple comparison experiments to be made between different molecular species.

\section{ACKNOWLEDGEMENTS}

This work was supported by EPSRC programme Grant No. EP/I032517/1 and ERC ASTEX Project No. 290467. A.S.J. and D.A.W. also acknowledge support from Marie Curie ITN EC317232, A.S.J. acknowledges support from NSERC PGSD3-454096-2014 and D.R.A. acknowledges support from the Imperial College JRF scheme. MC thanks support from Project CTQ2013-43086-P (MINECO, Spain)

\footnotetext{
${ }^{1}$ Franck Lépine, Misha Y. Ivanov, and Marc J. J. Vrakking. Attosecond molecular dynamics: fact or fiction? Nature Photonics, 8(3):195204, feb 2014.

${ }^{2}$ F Calegari, D Ayuso, A Trabattoni, L Belshaw, S De Camillis, S Anumula, F Frassetto, L Poletto, A Palacios, P Decleva, J B Greenwood, F Martín, and M Nisoli. Ultrafast electron dynamics in phenylalanine initiated by attosecond pulses. Science, 346(6207):336-9, 2014.

${ }^{3}$ Martin Schultze, Elisabeth M. Bothschafter, Annkatrin Sommer, Simon Holzner, Wolfgang Schweinberger, Markus Fiess, Michael Hofstetter, Reinhard Kienberger, Vadym Apalkov, Vladislav S. Yakovlev, Mark I. Stockman, and Ferenc Krausz. Controlling dielectrics with the electric field of light. Nature, 493(7430):75-78, 2012.

${ }^{4}$ Yoann Pertot, Cédric Schmidt, Mary Matthews, Adrien Chauvet, Martin Huppert, Vit Svoboda, Aaron von Conta, Andres Tehlar,
} 
Denitsa Baykusheva, Jean-pierre Wolf, and Hans Jakob Wörner. Time-resolved x-ray absorption spectroscopy with a water window high-harmonic source. Science, 355(6322):264-267, jan 2017.

${ }^{5}$ Daniel M Neumark. TIME-RESOLVED PHOTOELECTRON SPECTROSCOPY OF MOLECULES AND CLUSTERS. Annual Review of Physical Chemistry, 52(1):255-277, oct 2001.

${ }^{6}$ Martin Pitzer, Maksim Kunitski, Allan S Johnson, Till Jahnke, Hendrik Sann, Felix Sturm, Lothar Ph. H Schmidt, Horst SchmidtBöcking, Reinhard Dörner, Jürgen Stohner, Julia Kiedrowski, Michael Reggelin, Sebastian Marquardt, Alexander Schießer, Robert Berger, and Markus S Schöffler. Direct Determination of Absolute Molecular Stereochemistry in Gas Phase by Coulomb Explosion Imaging. Science, 341(6150):1096-1100, 2013.

${ }^{7}$ T. Kitamura, T. Nishide, H. Shiromaru, Y. Achiba, and N. Kobayashi. Direct observation of dynamic chirality by Coulomb explosion imaging. The Journal of Chemical Physics, 115(1):5, 2001.

${ }^{8} \mathrm{~J}$ P Marangos. Development of high harmonic generation spectroscopy of organic molecules and biomolecules. Journal of Physics B: Atomic, Molecular and Optical Physics, 49(13):132001, 2016.

${ }^{9} \mathrm{~F}$ Frank, C Arrell, T Witting, W a Okell, J McKenna, J S Robinson, C a Haworth, D Austin, H Teng, I a Walmsley, J P Marangos, and J W G Tisch. Invited review article: technology for attosecond science. The Review of scientific instruments, 83(7):071101, jul 2012.

${ }^{10} \mathrm{R}$ Locher, M Lucchini, J Herrmann, M Sabbar, M Weger, a Ludwig, L Castiglioni, M Greif, M Hengsberger, L Gallmann, and U Keller. Versatile attosecond beamline in a two-foci configuration for simultaneous time-resolved measurements. The Review of scientific instruments, 85(1):013113, jan 2014.

${ }^{11}$ S Baker, J. S. Robinson, C. A. Haworth, H Teng, R. A. Smith, C. C. Chirila, M. Lein, J. W. G. Tisch, and J. P. Marangos. Probing Proton Dynamics in Molecules on an Attosecond Time Scale. Science, 312(5772):424-427, apr 2006.

${ }^{12}$ Olga Smirnova, Yann Mairesse, Serguei Patchkovskii, Nirit Dudovich, David Villeneuve, Paul Corkum, and Misha Yu Ivanov. High harmonic interferometry of multi-electron dynamics in molecules. $\mathrm{Na}$ ture, 460(7258):972-977, 2009.

${ }^{13}$ Hans Jakob Worner, Hiromichi Niikura, Julien B. Bertrand, P. B. Corkum, and D. M. Villeneuve. Observation of electronic structure minima in high-harmonic generation. Physical Review Letters, 102(10):1-4, 2009.

${ }^{14}$ S. Baker, J. S. Robinson, M. Lein, C. C. Chiril, R. Torres, H. C. Bandulet, D. Comtois, J. C. Kieffer, D. M. Villeneuve, J. W. G. Tisch, and J. P. Marangos. Dynamic Two-Center Interference in High-Order Harmonic Generation from Molecules with Attosecond Nuclear Motion. Physical Review Letters, 101(5):053901, jul 2008.

${ }^{15}$ P. M. Kraus, O. I. Tolstikhin, D. Baykusheva, a. Rupenyan, J. Schneider, C. Z. Bisgaard, T. Morishita, F. Jensen, L. B. Madsen, and H. J. Wörner. Observation of laser-induced electronic structure in oriented polyatomic molecules. Nature Communications, 6(May):7039, 2015. ${ }^{16}$ R. Cireasa, a. E. Boguslavskiy, B. Pons, M. C. H. Wong, D. Descamps, S. Petit, H. Ruf, N. Thiré, A. Ferré, J. Suarez, J. Higuet, B. E. Schmidt, a. F. Alharbi, F. Légaré, V. Blanchet, B. Fabre, S. Patchkovskii, O. Smirnova, Y. Mairesse, and V. R. Bhardwaj. Probing molecular chirality on a sub-femtosecond timescale. Nature Physics, 11(8):654-658, jun 2015.

${ }^{17}$ Shambhu Ghimire, Anthony D. DiChiara, Emily Sistrunk, Pierre Agostini, Louis F. DiMauro, and David a. Reis. Observation of high-order harmonic generation in a bulk crystal. Nature Physics, $7(2): 138-141$, dec 2010.

${ }^{18}$ P. Heissler, E. Lugovoy, R Hörlein, L. Waldecker, J. Wenz, M. Heigoldt, K. Khrennikov, S. Karsch, F. Krausz, B. Abel, and G. D. Tsakiris. Using the third state of matter: high harmonic generation from liquid targets. New Journal of Physics, 16(11):113045, nov 2014.

${ }^{19}$ Mette B Gaarde, Jennifer L Tate, and Kenneth J Schafer. Macroscopic aspects of attosecond pulse generation. Journal of Physics B: Atomic, Molecular and Optical Physics, 41(13):132001, jul 2008.

${ }^{20}$ B. P. Wilson, K. D. Fulfer, S. Mondal, X. Ren, J. Tross, E. D. Poliakoff, J. Jose, Anh-Thu Le, R. R. Lucchese, and C. Trallero-Herrero. High order harmonic generation from SF6: Deconvolution of macroscopic effects. The Journal of Chemical Physics, 145(22):224305, 2016.

${ }^{21} \mathrm{~S}$ Haessler, J Caillat, and P Salières. Self-probing of molecules with high harmonic generation. Journal of Physics B: Atomic, Molecular and Optical Physics, 44(20):203001, 2011.

${ }^{22} \mathrm{~N}$ Hay, R. de Nalda, T Halfmann, K.J. Mendham, M.B. Mason, M Castillejo, and J.P. Marangos. High-order harmonic generation from organic molecules in ultra-short pulses. The European Physical Journal D, 14(2):231-240, may 2001.

${ }^{23}$ Uzi Even and Nachum A. Lavie. Even Lavie Valve - Website.

${ }^{24}$ U Even, J Jortner, D Noy, N Lavie, and C Cossart-Magos. Cooling of large molecules below $1 \mathrm{~K}$ and He clusters formation. Journal of Chemical Physics, 112(18):8068-8071, 2000.

${ }^{25}$ N. Hay, M. Castillejo, R. de Nalda, E. Springate, K. J. Mendham, and J. P. Marangos. High-order harmonic generation in cyclic organic molecules. Physical Review A, 61(5):053810, apr 2000.

${ }^{26}$ N. G. Korobeishchikov, a. E. Zarvin, V. V. Kalyada, and a. a. Schmakov. Pulsed Gas Jets for Formation of High-Intensity Cluster Beams. Advances in Materials Physics and Chemistry, 02(December):31-34, 2012.

${ }^{27}$ Henrik Stapelfeldt and Tamar Seideman. Aligning molecules with strong laser pulses. Rev. Mod. Phys., 75(2):543-557, 2003.

${ }^{28}$ C. Vozzi, M. Nisoli, J-P. Caumes, G. Sansone, S. Stagira, S. De Silvestri, M. Vecchiocattivi, D. Bassi, M. Pascolini, L. Poletto, P. Villoresi, and G. Tondello. Cluster effects in high-order harmonics generated by ultrashort light pulses. Applied Physics Letters, 86(11):111121, mar 2005. 